\title{
Diatomáceas (Bacillariophyceae) perifíticas em substratos naturais do rio das Pedras, município de Guarapuava, Paraná, Brasil
}

\section{Periphytic diatoms (Bacillariophyceae) in natural substrates from the rio das Pedras, located in Guarapuava, state of Paraná, Brazil}

\author{
Gisele Carolina Marquardt \\ Cynthia Beatriz Furstenberger \\ Tiago Elias Chaouiche \\ Rebeca Caparica \\ Universidade Estadual do Centro-Oeste \\ Luciana Carapunarla \\ Universidade Estadual de Maringá
}

\begin{abstract}
Resumo: Este trabalho é parte do estudo sobre as diatomáceas perifíticas presentes no rio das Pedras, Guarapuava, no estado do Paraná, Brasil, e resultou na identificação de 35 táxons infragenéricos, distribuídos em 12 gêneros e seis famílias. Foram considerados táxons constantes: Frustulia saxonica Rabenhorst, Gomphonema gracile Ehrenberg e Gomphonema parvulum (Kützing) Kützing. A análise da comunidade diatomológica forneceu informações das condições ecológicas, sendo que a predominância de espécies eutróficas revela águas ricas em substâncias nutritivas, indicando que o sistema encontrase alterado devido ao efeito causado por início de degradação de compostos orgânicos lançados no corpo d'água.
\end{abstract}

Palavras-chave: Bacillariophyceae. Diatomáceas. Perifíton. Rio das Pedras.

Abstract: This work is part of the study of periphytic diatoms in the rio das Pedras, located in Guarapuava, state of Paraná, Brazil, which resulted in the identification of 34 infrageneric taxa distributed in 12 genera and six families. The following taxa were considered frequent: Frustulia saxonica Rabenhorst, Gomphonema gracile Ehrenberg, and Gomphonema parvulum (Kützing) Kützing. This diatomological analysis provided information on the ecological conditions of the river, while the predominance of eutrophic species reveals that its waters are rich with nutritive substances. This indicates that the system is altered due to effect of the beginning of degradation of organic compounds degradation thrown in the river.

Keywords: Bacillariophyceae. Diatoms. Periphyton. Rio das Pedras. 


\section{INTRODUÇÃO}

No Paraná, o estudo das diatomáceas (Bacillariophyceae) perifíticas tem recebido atenção por vários autores, os quais vêm realizando tanto trabalhos de cunho ecológico quanto estudos taxonômicos e florísticos. Tais trabalhos vêm sendo desenvolvidos principalmente nos reservatórios paranaenses, segundo, por exemplo, os estudos de Cetto et al. (2004), que analisaram a comunidade de algas perifíticas em diferentes estações do ano (verão e inverno) no reservatório de Irai. Já Brassac e Ludwig (2005) realizaram o levantamento florístico das diatomáceas dos rios da área de abrangência do reservatório da Usina Hidrelétrica de Salto Caxias. Ferrari e Ludwig (2007), por sua vez, verificaram a flórula das Coscinodiscophyceae, Fragilariophyceae e Bacillariophyceae (Achnanthales) do rio Ivaí. Há trabalhos parcialmente ou ainda não publicados.

Ecossistemas lóticos apresentam um fluxo de água contínuo unidirecional, ausência de estratificação térmica e alta variação das condições físicas e químicas, devido à presença característica da correnteza. Apresentam, ainda, variações estruturais ao longo do seu percurso, provocando a formação de diversos tipos de ambientes, desde locais rasos até com grandes profundidades. A erosão é uma característica mais presente em ambientes de águas correntes do que em ambientes lênticos, tornando mais intenso o fluxo de materiais e nutrientes. (KRUPEK, 2006). Comunidades perifíticas, ou simplesmente perifíton, são utilizadas para definir a complexa comunidade que se encontra aderida ao substrato, incluindo uma grande quantidade de microalgas e outros organismos não algais. A importância do perifíton como indicador da qualidade da água e de seu estado trófico foi evidenciada por vários pesquisadores, principalmente para rios e pequenos cursos d'água. (SLÁDECEK; SLÁDEKOVÁ, 1963; SLÁDECEK, 1969; PIECZYNSKA, 1970; SLÁDECKOVA; SLÁDECEK, 1977; SLÁDECKOVÁ et al.,1983; WATANABE et al., 1985, 1986 ab; WATANABE, 1990; SLÁDECKOVÁ, $1994 \mathrm{ab}$ ). As diatomáceas, dentre os demais grupos de algas, constituem uma parcela significativa nessa comunidade, principalmente em ambientes lóticos, pois apresentam adaptações que favorecem a fixação aos substratos, além de serem consideradas colonizadoras primárias. (LEANDRINI, 1999). O presente trabalho propõe a avaliação qualitativa das comunidades de diatomáceas para futura confecção de parâmetros ecológicos em um dado percurso do rio das Pedras, no município de Guarapuava, Paraná.

\section{MATERIAL E MÉTODOS}

A bacia do rio das Pedras possui aproximadamente $330 \mathrm{~km}^{2}$ entre as latitudes $25^{\circ} 13^{\prime} 10^{\prime \prime} \mathrm{S}$ e $25^{\circ} 26^{\prime} 24^{\prime \prime} \mathrm{S}$ e longitudes $51^{\circ} 13^{\prime} 10^{\prime \prime} \mathrm{W}$ e $51^{\circ} 28^{\prime} 40^{\prime \prime} \mathrm{W}$. É a principal fonte de abastecimento do município de Guarapuava. (SANTOS; KOBIYAMA, 2003). Distinguem-se, quanto ao uso da terra na bacia do rio das Pedras, três grandes unidades (BATTISTELLI et al., 2004): a primeira no curso inferior, caracterizada por uma paisagem heterogênea e grande diversidade de uso da terra em pequenas extensões de áreas (por exemplo, agricultura, indústria, área urbana, etc.); uma segunda, no curso superior da bacia, engloba as áreas pertencentes a APA da Serra da Esperança e às principais nascentes do rio das Pedras, predominando ali uma paisagem homogênea, com grandes extensões de áreas florestadas; e uma 
terceira região, localizada no curso médio do rio, representada por uma paisagem na qual se intercalam pequenas e grandes áreas utilizadas para o desenvolvimento de reflorestamento/florestamento com plantas exóticas.

As amostras de água foram coletadas de março a novembro de 2004, num percurso aproximado de $35 \mathrm{~km}$ do rio das Pedras, onde foram demarcados 36 pontos com distâncias variando entre $500 \mathrm{~m}$ e $1.000 \mathrm{~m}$. As amostras para o estudo qualitativo foram obtidas através de espremido e fragmentação manual de partes de vegetais submersos, contendo mucilagem aderida e coleta das algas circundantes ao vegetal submerso com rede de plâncton com abertura de malha de 20 $\mu \mathrm{m}$. Empregou-se como fixador formalina a $2 \%$. O exame qualitativo do material em microscópio ótico binocular baseou-se em duas séries de lâminas permanentes, uma com material não oxidado e outra com amostras submetidas à oxidação (SIMONSEN 1974, modificada por MOREIRA FILHO; VALENTE-MOREIRA 1981). O enquadramento taxonômico seguiu Round, Crawford e Mann (1990). A identificação de gêneros, espécies, variedades e formas taxonômicas baseouse em Hustedt (1927-1930, 1930, 1939, 1952, 1965, 1961-1966), Patrick e Reimer (1966, 1975) e Krammer e Lange-Bertalot (1986, 1988, 1991a, 1991b). As amostras e lâminas permanentes encontram-se no Herbário da Universidade Estadual do Centro-Oeste (UNICENTRO).

\section{RESULTADOS}

\section{Divisão Bacillariophyta}

Classe Bacillariophyceae

Subclasse Bacillariophycidae

Ordem Cymbellales

Família Cymbellaceae

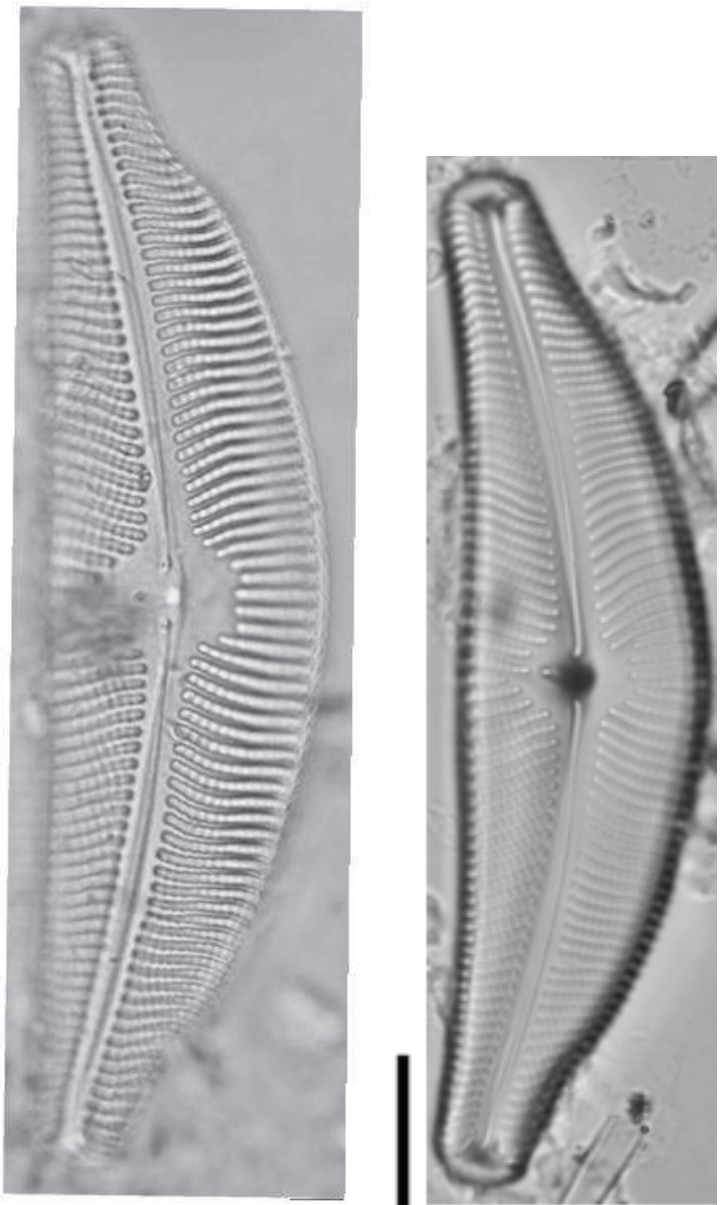

Figura 1 - Cymbella tumida (Brébisson ex Kützing) Van Heurck var. tumida Syn. Diat. Belg., p. 64, pl. 2, fig. 10. 1880-85.

Valva dorsiventral; margem dorsal convexa, margem ventral levemente côncava, extremidades atenuadotruncadas; área axial arqueada; área central orbicular; rafe ao longo da linha mediana da valva, curvada; estigma ventral presente, avançando para a porção dorsal da área central; 10-11 estrias unisseriadas em 10 $\mu \mathrm{m}$, com aréolas alargadas, grosseiras, 14-15 em $10 \mu \mathrm{m}$; eixo apical: 48,5-77,4 $\mu \mathrm{m}$; eixo transapical: 15-19,6 $\mu \mathrm{m}$.

Material examinado: BRASIL. Paraná: Guarapuava, rio das Pedras, 26/III/2004, Bergamaschi, A.C. E Kawa, M. (RP006, RP007, RP008, RP009, RP010, RP012, RP015, RP018); Guarapuava, rio das 
Pedras, 30/IV/2004, Bergamaschi, A.C. E Kawa, M. (RP046, RP054); Guarapuava, rio das Pedras, 05/V/2004, Bergamaschi, A.C. $\mathcal{E}$ Kawa, M. (RP107); Guarapuava, rio das Pedras, 18/VI/2004, Bergamaschi, A.C. E Kawa, M. (RP113, RP116, RP118, RP120, RP123, RP131); Guarapuava, rio das Pedras, 09/VIII/2004, Bergamaschi, A.C. \& Kawa, M. (RP150, RP151, RP152, RP166).

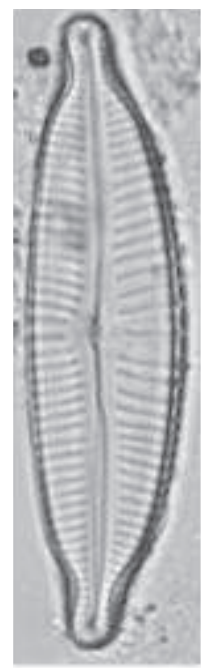

Figura 2 - Cymbopleura naviculiformis (Auerswald ex Heiberg) K. Krammer

Diatoms Europe: 56; pl. 76, figs. 1-13; pl. 77, figs. 1-13; pl. 78, figs. 1-8; pl. 79: figs. 1-14; pl. 80: figs. 12; pl. 82: figs.1-12; pl. 83: figs. 9-11. 2003.
Valva dorsiventral, lanceolada; margem dorsal convexa, margem ventral moderadamente convexa; extremidades rostradas a rostrado-subcapitadas; área axial estreita, linear; área central orbicular; rafe levemente arqueada, fissuras terminais dorsalmente fletidas; 12 16 estrias transapicais em $10 \mu \mathrm{m}$, radiadas, relativamente mais afastadas umas das outras na região mediana da valva; eixo apical: 29,6-33,6 $\mu \mathrm{m}$; eixo transapical: 8-9,3 $\mu \mathrm{m}$.

Material examinado: BRASIL. Paraná: Guarapuava, rio das Pedras, 26/ III/2004, Bergamaschi, A.C. E Kawa, M. (RP002, RP004, RP009, RP011, RP012, RP013, RP015, RP016); Guarapuava, rio das Pedras, 30/IV/2004, Bergamaschi, A.C. \& Kawa, M. (RP039, RP040, RP043, RP048, RP057); Guarapuava, rio das Pedras, 05/V/2004, Bergamaschi, A.C. E Kawa, M. (RP105); Guarapuava, rio das Pedras, 18/VI/2004, Bergamaschi, A.C. E Kawa, M. (RP110, RP113, RP114, RP115, RP118, RP119, RP127, RP128, RP130, RP132, RP137, RP141); Guarapuava, rio das Pedras, 09/VIII/2004, Bergamaschi, A.C. E Kawa, M. (RP144, RP145, RP148, RP149, RP153, RP158).

\section{Encyonema Kützing}

Chave para os táxons do Gênero Encyonema

1. Extremidades valvares atenuado-arredondadas ................................................................ 2

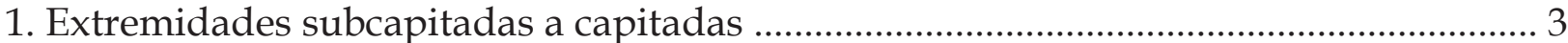

2. Extremidades subcapitadas Encyonema perpusilum

2. Extremidades capitadas

3. Aréolas inconspícuas Encyonema angustecapitatum

3. Aréolas conspícuas Encyonema sp

4. Extremidades fletidas ventralmente 4

4. Extremidades retas 5

5. Área axial alargando-se formando área central pequena Encyonema silesiacum

5. Área axial alargando-se formando área central mais expandida neomesianum 


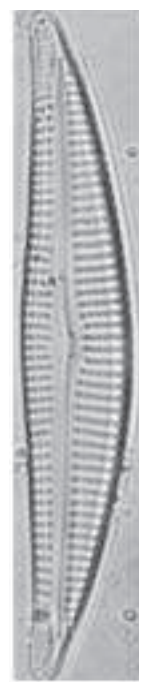

Figura 3 - Encyonema neogracile Krammer Bibl. Diatomol., v. 36, p. 177-178, pl. 82, figs. 1-7, 12-13, 1997.

Valva dorsiventral; margem dorsal convexa, margem ventral levemente convexa; extremidades atenuadoarredondadas; área axial estreita, linear, alargando-se na região mediana formando área central pequena; rafe filiforme, excêntrica, aproximadamente central, com extremidades proximais dorsalmente curvadas e extremidades distais em forma de vírgula, curvadas ventralmente; estigmoide presente na extremidade da estria mediana dorsal, 10-14 estrias transapicais em $10 \mu \mathrm{m}$, pontuadas, paralelas; cerca de sete aréolas em $10 \mu \mathrm{m}$; eixo apical: 36-68,8 $\mu \mathrm{m}$; eixo transapical: 7,4-13,2 $\mu \mathrm{m}$.

Material examinado: BRASIL. Paraná: Guarapuava, rio das Pedras, 26/III/2004, Bergamaschi, A.C. E Kawa, M. (RP001, RP002, RP004, RP007, RP008, RP009, RP010, RP011, RP014, RP016, RP018, RP019, RP020, RP023, RP024); Guarapuava, rio das Pedras, 30/IV/2004, Bergamaschi, A.C. E Kawa, M. (RP044, RP046, RP054, RP057); Guarapuava, rio das Pedras, 05/V/2004, Bergamaschi, A.C. E Kawa, M. (RP104, RP107, RP108); Guarapuava, rio das Pedras, 18/VI/2004, Bergamaschi,
A.C. E Kawa, M. (RP110, RP112, RP113, RP118, RP119, RP120, RP123, RP127, RP128, RP130, RP131, RP132, RP133, RP137, RP138, RP139, RP141, RP142); Guarapuava, rio das Pedras, 09/VIII/2004, Bergamaschi, A.C. E Kawa, M. (RP144, RP146, RP149, RP150, RP152, RP157, RP158, RP159, RP185).

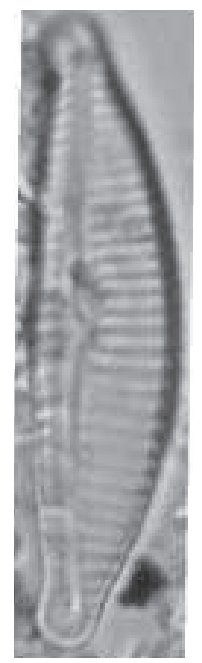

Figura 4 - Encyonema augustecapitatum Krammer Biblioth. Diatomol., v. 37, p. 192, pl. 130, figs. 8-15, 1997.

Valvas dorsiventrais; margem dorsal convexa; margem ventral levemente convexa; extremidades capitadas; área axial linear, estreita com área central pequena, irregular; rafe excêntrica, filiforme com extremidades distais ventralmente fletidas; extremidades proximais dorsalmente fletidas, dilatadas em poro; estrias dorsais e ventrais paralelas na região mediana, tornando-se levemente radiadas no sentido das extremidades; 13 estrias dorsais em 10 $\mu \mathrm{m}$; Aréolas inconspícuas. Eixo apical: 23,5 $\mu \mathrm{m}$; eixo transapical: 5,7 $\mu \mathrm{m}$.

Material examinado: BRASIL. Paraná: Guarapuava, rio das Pedras, 26/III/2004, Bergamaschi, A.C. E Kawa, M. (RP024). 


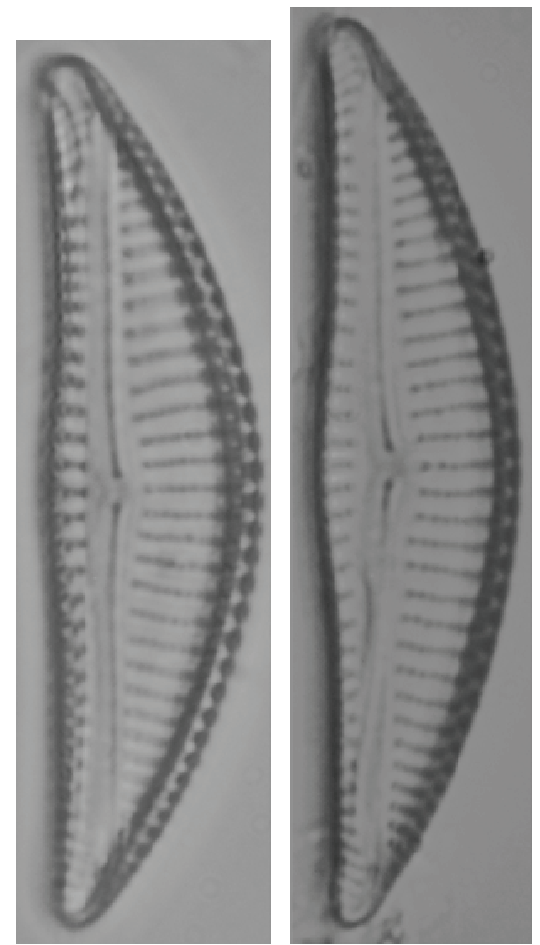

Figura 5 - Encyonema neomesianum Krammer Biblioth. Diatomol., v. 36, pt. 1, p. 84, pl. 40, figs. 6-9, pl. 54, figs. 6-7, pl. 99, figs. 4-7, 1997.

Valva dorsiventral, margem dorsal convexa; margem ventral levemente convexa, apresentando intumescência mediana; extremidades atenuadoarredondadas, ventralmente fletidas; área axial linear, alargando-se na região mediana para formar a área central mais expandida para o lado dorsal; rafe excêntrica, extremidades proximais dorsalmente fletidas, dilatadas em poro; fissuras terminais da rafe em forma de vírgula, ventralmente fletidas; estigmoide presente na extremidade da estria mediana dorsal. 10-12 estrias transapicais em $10 \mu \mathrm{m}$, pararelas na região mediana a levemente radiadas nas extremidades; eixo apical: 30,6-63,5 $\mu \mathrm{m}$; eixo transapical: 8,1-14,1 $\mu \mathrm{m}$.

Material examinado: BRASIL. Paraná: Guarapuava, rio das Pedras, 26/ III/2004, Bergamaschi, A.C. E Kawa, M. (RP002, RP004, RP005, RP006, RP007, RP008, RP009, RP011 RP012, RP013, RP014, RP15, RP016, RP018, RP020, RP021, RP022,
RP024); Guarapuava, rio das Pedras, 30/ IV/2004, Bergamaschi, A.C. E Kawa, M. (RP038, RP040, RP041, RP043, RP046, RP051); Guarapuava, rio das Pedras, 05/V/2004, Bergamaschi, A.C. E Kawa, M. (RP097, RP096); Guarapuava, rio das Pedras, 18/VI/2004, Bergamaschi, A.C. E Kawa, M. (RP110, RP112, RP116, RP118, RP119, RP122, RP124, RP125, RP127, RP128, RP129, RP131, RP135, RP136); Guarapuava, rio das Pedras, 09/VIII/2004, Bergamaschi, A.C. E Kawa, M. (RP144, RP145, RP146, RP150, RP158).
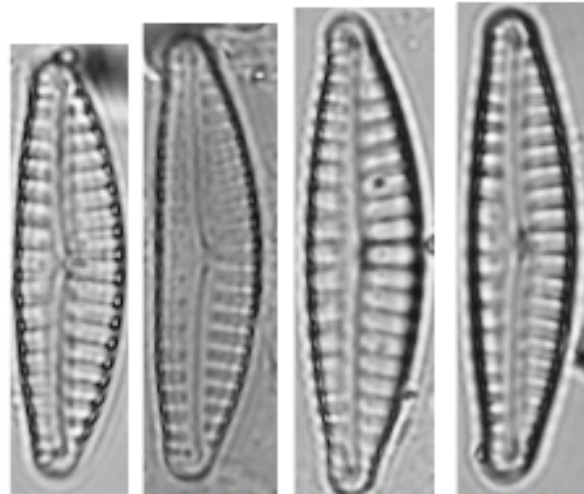

Figura 6 - Encyonema perpusilum (A. Cleve) D.G. Mann Round, Crawford \& Mann. The Diatoms, p. 667. 1990.

Valva dorsiventral, margem dorsal convexa; margem ventral levemente convexa; extremidades atenuadas, subcapitadas, área axial estreita, linear; área central pequena, circular; rafe reta, excêntrica, apresentando extremidades proximais voltadas para a margem dorsal; 9-13 estrias paralelas em $10 \mu \mathrm{m}$, equidistantes; eixo apical 19,3-22 $\mu \mathrm{m}$; eixo transapical: 4,8-7,1 $\mu \mathrm{m}$.

Material examinado: BRASIL. Paraná: Guarapuava, rio das Pedras, 26/III/2004, Bergamaschi, A.C. E Kawa, M. (RP002, RP007, RP010); Guarapuava, rio das Pedras, 30/IV/2004, Bergamaschi, A.C. E Kawa, M. (RP040 RP041); Guarapuava, rio das Pedras, 05/V/2004, Bergamaschi, A.C. \& Kawa, M. (RP104, RP107, RP108); Guarapuava, rio das Pedras, 18/VI/2004, 
Bergamaschi, A.C. E Kawa, M. (RP115, RP135, RP136, RP137, RP141, RP142); Guarapuava, rio das Pedras, 09/VIII/2004, Bergamaschi, A.C. E Kawa, M. (RP145, RP150, RP158, RP185).

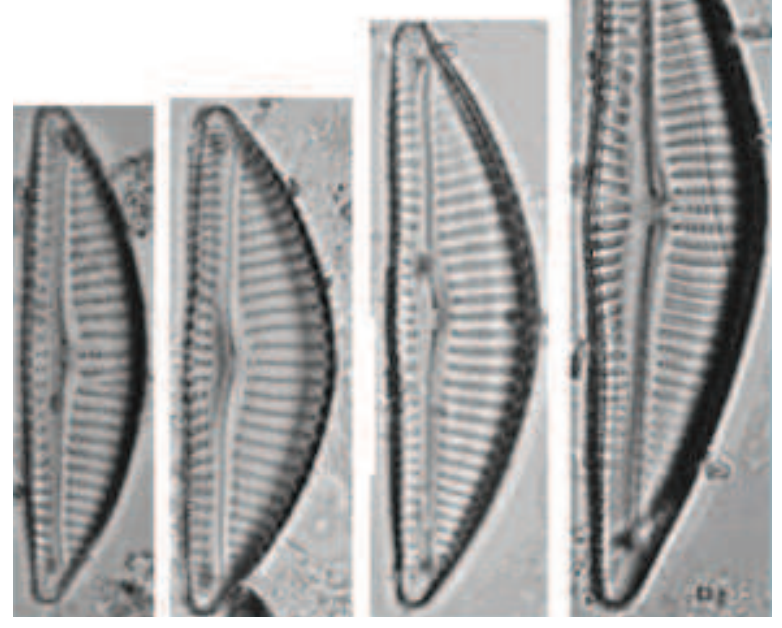

Figura 7 - Encyonema silesiacum (Bleisch) D.G. Mann Round, Crawford \& Mann. The Diatoms, p. 667. 1990.

Valva dorsiventral; margem dorsal convexa; margem ventral reta a côncava ou convexa, às vezes com leve intumescimento mediano; extremidades estreitas atenuadoarredondadas; área axial estreita; área central levemente expandida; rafe excêntrica, extremidades proximais dorsalmente fletidas, dilatadas em poro; fissuras terminais da rafe em forma de vírgula, ventralmente fletidas; estigmoide presente na extremidade da estria dorsal; estrias dorsais e ventrais paralelas, tornando-se radiadas a convergentes no sentido das extremidades. Aréolas conspícuas, 25 a 30 em $10 \mu \mathrm{m}$. 10-12 estrias paralelas em $10 \mu \mathrm{m}$, equidistantes; eixo apical 30,6-51,2 $\mu \mathrm{m}$; eixo transapical: 8,1$11,8 \mu \mathrm{m}$.

Material examinado: BRASIL. Paraná: Guarapuava, rio das Pedras, 26/III/2004, Bergamaschi, A.C. E Kawa, M. (RP002,
RP007, RP010); Guarapuava, rio das Pedras, 30/IV/2004, Bergamaschi, A.C. E Kawa, M. (RP040 RP041); Guarapuava, rio das Pedras, 05/V/2004, Bergamaschi, A.C. E Kawa, M. (RP104, RP108); Guarapuava, rio das Pedras, 18/VI/2004, Bergamaschi, A.C. E Kawa, M. (RP115, RP135, RP136, RP137, RP141); Guarapuava, rio das Pedras, 09/VIII/2004, Bergamaschi, A.C. \& Kawa, M. (RP145, RP150, RP185).

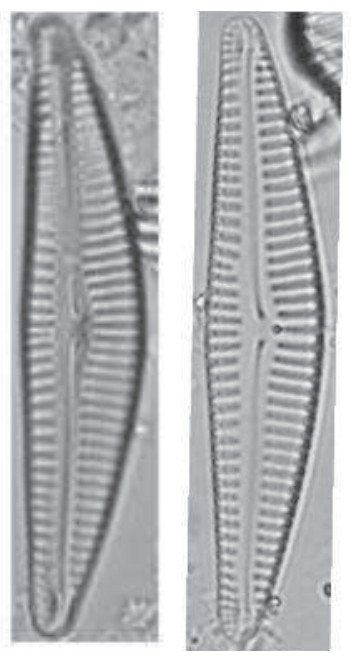

Figura 8 - Encyonema sp

Valva dorsiventral; margem dorsal convexa; margem ventral levemente convexa; extremidades atenuadoarredondadas; área axial linear, estreita; alargando-se na região mediana, mais expandida ventralmente; rafe filiforme, excêntrica, ventral; estigmoide presente na extremidade da estria mediana dorsal; estrias dorsais levemente radiadas, estrias ventrais paralelas a convergentes em direção às extremidades, 11-12 estrias dorsais em $10 \mu \mathrm{m}$.; aréolas inconspícuas; eixo apical: $36-41,4 \mu \mathrm{m}$; eixo transapical: 7,6-8,4 $\mu \mathrm{m}$.

Material examinado: BRASIL. Paraná: Guarapuava, rio das Pedras, 30/IV/2004, Bergamaschi, A.C. E Kawa, M. (RP044, RP046); Guarapuava, rio das Pedras, 09/ VIII/2004, Bergamaschi, A.C. E Kawa, M. (RP144, RP146). 


\section{Encyonopsis Krammer}

Chave para os táxons do Gênero Encyonopsis

1. Extremidades sub-rostradas

Encyonopsis kaingensis

1. Extremidades rostradas Encyonopsis sp

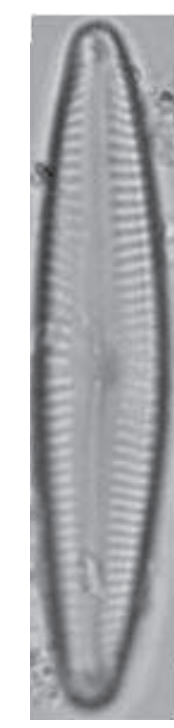

Figura 9 - Encyonopsis kaingensis (Vyverman) Krammer Bib. Diat., n.37, t.2, p.139, pl. 140, fig.17-19. 1997

Valvas levemente assimétricas, lineares; margens dorsal e ventral convexas; extremidades sub-rostradas; área axial lanceolada; área central lanceolada; rafe levemente sinuosa, terminações distais ventralmente fletidas, terminações proximais dorsalmente fletidas; estigmoide ausente; 16 estrias radiadas em $10 \mu \mathrm{m}$, convergentes no sentido das extremidades valvares; aréolas inconspícuas;eixo apical: 31,4 $\mu \mathrm{m}$; eixo transapical: 5,7 $\mu \mathrm{m}$.

Material examinado: BRASIL. Paraná: Guarapuava, rio das Pedras, 26/III/2004, Bergamaschi, A.C. E Kawa, M. (RP010)

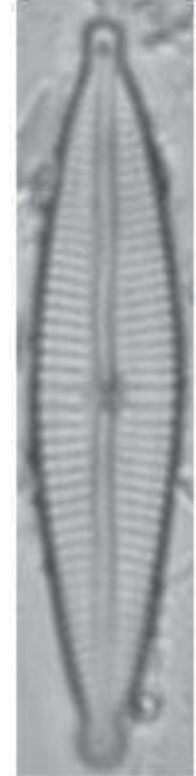

Figura 10 -Encyonopsis $s p$

Valvaslanceoladas com dorsiventralidade pouco pronunciada; margem dorsal e ventral quase igualmente convexas; extremidades capitadas; área axial linear, estreita abrindo em área central pouco expandida, elíptica, limitada pelo encurtamento irregular das estrias medianas; rafe sinuosa, filiforme; 16 estrias em $10 \mu \mathrm{m}$; paralelas na região mediana tornando-se levemente convergentes no sentido das extremidades. Aréolas inconspícuas. Eixo apical: 29,2 $\mu \mathrm{m}$; eixo transapical: $6,4 \mu \mathrm{m}$;

Material examinado: BRASIL. Paraná: Guarapuava, rio das Pedras, 30/IV/2004, Bergamaschi, A.C. E Kawa, M. (RP046);

Comentários: o táxon observado é semelhante à Encyonopsis frequentis Krammer, no entanto, a área central e estrias centrais dessa última formam uma estrutura cruciforme característica da espécie (KRAMMER, 1997), o que não pode ser observado no material estudado. 
Placoneis C. Mereschkowsky

1. Presença de estigma Placoneis disparilis

1. Ausência de estigma Placoneis exigua
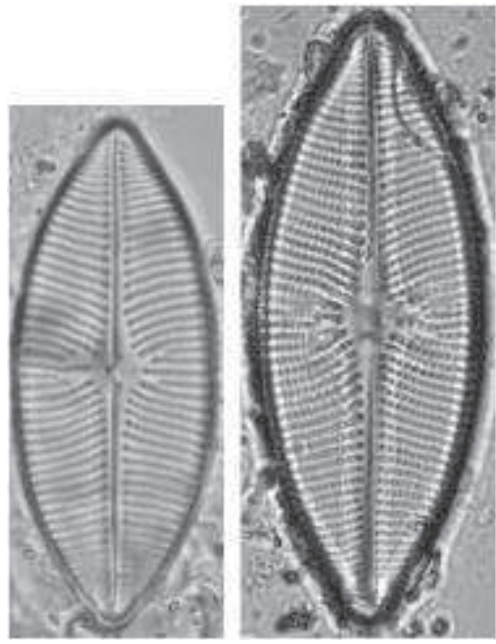

Figura 11 - Placoneis disparilis (Hustedt) Metzeltin \& Lange-Bertalot

Iconogr. Diatomol. 5, p. 197. 1998.

Valva elíptico-lanceolada; extremidades levemente rostradas. Rafe linear reta apresentando extremidades proximais dilatadas em nódulos. Área central irregular apresentando um poro isolado. 14-15 estrias transapicais em $10 \mu \mathrm{m}$, radiadas com pontuações nítidas, 14 a 16 em $10 \mu \mathrm{m}$; eixo apical: 34-51,32 $\mu \mathrm{m}$; eixo transapical: 13-17,76 $\mu \mathrm{m}$.

Material examinado: BRASIL. Paraná: Guarapuava, rio das Pedras, 26/III/2004, Bergamaschi, A.C. E Kawa, M. (RP002, RP018, RP020); Guarapuava, rio das Pedras, 05/V/2004, Bergamaschi, A.C. E Kawa, M. (RP110); Guarapuava, rio das Pedras, 09/VIII/2004, Bergamaschi, A.C. E Kawa, M. (RP145).

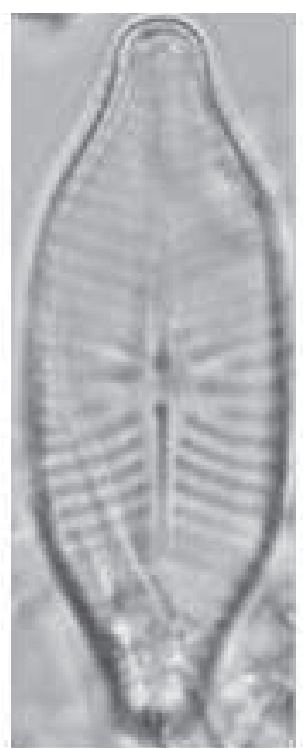

Figura 12 - Placoneis exigua (Gregory) Mereschkowsky Beih. Bot. Centr., v. 15, p. 13, pl. 1, fig. 17, 1903.

Valva linear-lanceolada; extremidades sub-rostradas; área axial linear, estreita; área central arredondada limitada por uma estria mediana alongada entre duas menores, estigma ausente; rafe filiforme, extremidades proximais sutilmente fletidas; 14 estrias radiadas em $10 \mu \mathrm{m}$; aréolas inconspícuas. Eixo apical: 22,85 $\mu \mathrm{m}$; eixo transapical: 7,8 $\mu \mathrm{m}$.

Material examinado: BRASIL. Paraná: Guarapuava, rio das Pedras, 26/III/2004, Bergamaschi, A.C. E Kawa, M. (RP002) 


\section{Gomphonema Ehrenberg}

Família Gomphonemataceae

Chave para os táxons de Gomphonema

1. Valvas com intumescência mediana

1. Valvas sem intumescência mediana

2. Constrição pouco acentuada entre a região mediana e o ápice

2. Constrição bastante acentuada entre a região mediana e o ápice

3. Presença de estigma

Gomphonema laticollum

Gomphonema capitatum

3. Ausência de estigma

Gomphonema brasiliense

4. Área axial estreita

4. Área axial ampla

Gomphonema clevei var. inaequilongum

5. Areolação insconspícua

5. Areolação conspícua

Gomphonema turris

6. Área central unilateral 7

6. Área central retangular

Gomphonema lagenula

7. Rafe sinuosa 8

7. Rafe reta

Gomphonema paroulum

8. Extremidade apical capitada Gomphonema subtile

8. Extremidade apical de outro modo 9

9. Bases atenuadas Gomphonema pseudoaugur

9. Bases agudo-atenuadas e atenuado-arredondadas Gomphonema gracile

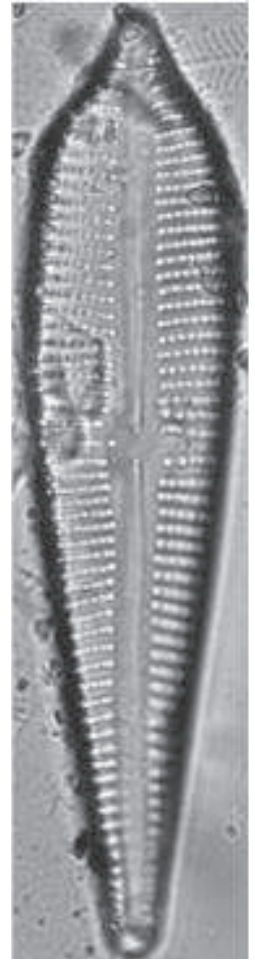

Figura 13 - Gomphonema turris Ehrenberg Phys. Abh. Akad. Wiss. Ber., p. 416, 1843.

Valva heteropolar clavada dilatada na metade superior; extremidade apical cuneada, sub-rostrada; extremidade basal arredondada. Rafe linear reta. Área central unilateral, formada pelo encurtamento de uma estria mediana. 11 estrias transapicais em $10 \mu \mathrm{m}$, paralelas, radiadas nas extremidades, pontuadas, 14 aréolas em $10 \mu \mathrm{m}$; eixo apical: 40-64,64 $\mu \mathrm{m}$;eixo transapical: 12-13 $\mu \mathrm{m}$.

Material examinado: BRASIL. Paraná: Guarapuava, rio das Pedras, 26/III/2004, Bergamaschi, A.C. E Kawa, M. (RP001, RP004, RP006, RP007, RP008, RP010, RP011, RP013, RP016, RP019, RP021, RP022, RP023); Guarapuava, rio das Pedras, 30/IV/2004, Bergamaschi, A.C. $\mathcal{E}$ Kawa, M. (RP039, RP048, RP050, RP053, RP057); Guarapuava, rio das Pedras, 05/V/2004, Bergamaschi, A.C. E Kawa, M. (RP108); Guarapuava, rio das Pedras, 18/VI/2004, Bergamaschi, A.C. E Kawa, M. (RP112, RP113, RP114, RP124, RP132); Guarapuava, rio das Pedras, 09/VIII/2004, Bergamaschi, A.C. E Kawa, M. (RP147, RP148, RP150, RP151, RP152, RP154, RP157, RP158, RP159). 


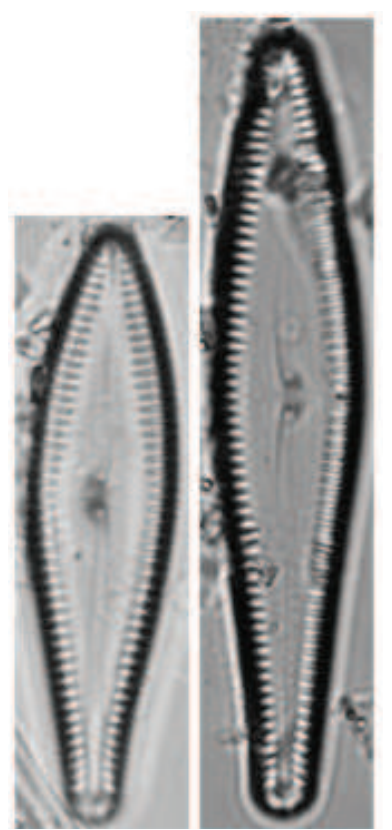

Figura 14 - Gomphonema brasiliense Grunow Naturw. Beitr. Kennth. Kauk., p. 110, 1879.

Valvas lanceoladas; heteropolares, ápices e bases atenuado-arredondadas. Área axial e área central amplamente lanceolado, formando área hialina quase atingindo as margens valvares; estigma ausente; rafe sinuosa, com extremidades distais fletidas para o lado oposto ao das extremidades proximais da rafe, extremidades proximais da rafe dilatadas em poro; 17 estrias em $10 \mu \mathrm{m}$, mais encurtadas na região mediana valvar, paralelas a levemente radiadas no sentido das extremidades; Aréolas inconspícuas. ; Eixo apical: 29,07- 39,09 $\mu \mathrm{m}$. Eixo transapical: 7,09-7,44 $\mu \mathrm{m}$;

Material examinado: BRASIL. Paraná: Guarapuava, rio das Pedras30/IV/2004, Bergamaschi, A.C. E Kawa, M. (RP037, RP042).

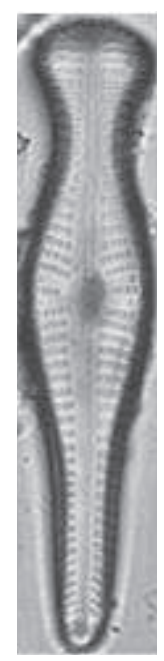

Figura 15 - Gomphonema capitatum Ehrenberg Infus., 217, pl. 18, fig. 2, 1838.

Valva clavada com intumescimento na região mediana. Extremidade apical amplamente arredondada; Extremidade basal arredondada. Esterno da rafe linear. Área central circular a elíptica, estigma na extremidade da estria mediana. Rafe levemente sinuosa. 10 estrias unisseriadas e radiadas em $10 \mu \mathrm{m}$, irregularmente encurtadas na região mediana, aréolas conspícuas, 18-20 em $10 \mu \mathrm{m}$; eixo apical: 37,6 $\mu \mathrm{m}$; eixo transapical: 8,57 $\mu \mathrm{m}$.

Material examinado: BRASIL. Paraná: Guarapuava, rio das Pedras, 26/III/2004, Bergamaschi, A.C. \& Kawa, M. (RP007).

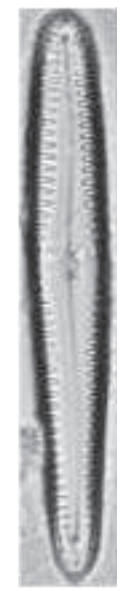

Figura 16 - Gomphonema clevei Fricke var. inaequilongum H. Kobayasi

J. Jap. Bot. 40: 350. f. 12a, b. 1965. 
Valva heteropolar, lanceolada; ápice arredondado, rafe sinuosa, presença de estigma em um dos lados da área central, hialina e amplamente lanceolada. 13 estrias grosseiras em $10 \mu \mathrm{m}$, restritas às margens, formando uma ampla área axial; eixo apical: 39,8 $\mu \mathrm{m}$; eixo transapical: 5,3 $\mu \mathrm{m}$.

Material examinado: BRASIL. Paraná: Guarapuava, rio das Pedras, 26/III/2004, Bergamaschi, A.C. E Kawa, M. (RP001, RP002, RP006, RP007, RP009, RP010, RP012, RP018); Guarapuava, rio das Pedras, 30/ IV/2004, Bergamaschi, A.C. E Kawa, M. (RP037, RP040, RP042, RP043, RP046, RP048, RP052, RP057); Guarapuava, rio das Pedras, 05/V/2004, Bergamaschi, A.C. $\mathcal{E}$ Kawa, M. (RP107, RP110); Guarapuava, rio das Pedras, 18/VI/2004, Bergamaschi, A.C. E Kawa, M. (RP112, RP113, RP114, RP116, RP117, RP127, RP128, RP130, RP131, RP136, RP138); Guarapuava, rio das Pedras, 09/VIII/2004, Bergamaschi, A.C. E Kawa, M. (RP146, RP147, RP147, RP148, RP149, RP150, RP151, RP157, RP158, RP187).

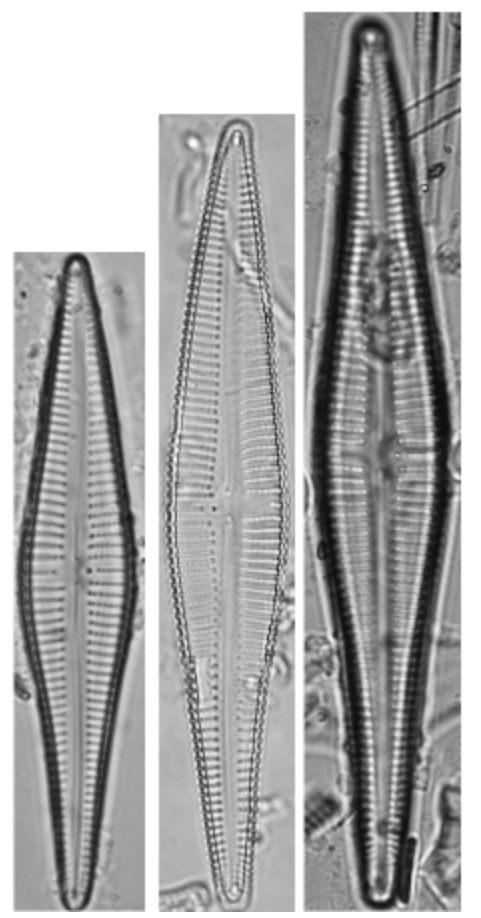

Figura 17 - Gomphonema gracile Ehrenberg Infusion., p. 27, pl. 18, fig. 3; Syn. Diat. Belg. 125, 1885.
Valva linear-lanceolada; extremidades apicais agudo-atenuadas atenuadasarredondadas e sub-rostradas, extremidades basais agudo-atenuadas e atenuado-arredondadas; área axial linear, estreita, área central unilateral formada pelo encurtamento da estria mediana, em sua extremidade no lado oposto ocorre um estigma; 11-12 estrias transapicais em $10 \mu \mathrm{m}$ levemente radiadas na porção mediana e radiadas em direção ao ápice e base; estrias medianas mais distantes das adjacentes entre si do que com as demais; rafe lateral, poros centrais distintos, levemente direcionados para o estigma; eixo apical 18,86-58,20 $\mu \mathrm{m}$; eixo transapical 4,92-8,73 $\mu \mathrm{m}$.

Material examinado: BRASIL. Paraná: Guarapuava, rio das Pedras, 26/III/2004, Bergamaschi, A.C. E Kawa, M. (RP006, RP007, RP008, RP009, RP011, RP017, RP018, RP022, RP023, RP024); Guarapuava, rio das Pedras, 30/IV/2004, Bergamaschi, A.C. $\mathcal{E}$ Kawa, M. (RP037, RP039, RP040, RP042, RP043, RP047, RP053, RP055, RP057); Guarapuava, rio das Pedras, 05/V/2004, Bergamaschi, A.C. E Kawa, M. (RP096, RP105, RP107, RP108); Guarapuava, rio das Pedras, 18/VI/2004, Bergamaschi, A.C. E Kawa, M. (RP110, RP112, RP113, RP114, RP116, RP117, RP118, RP122, RP123, RP124, RP125, RP126, RP127, RP128, RP130, RP131, RP132, RP135, RP136, RP137, RP139, RP141, RP142); Guarapuava, rio das Pedras, 09/VIII/2004, Bergamaschi, A.C. E Kawa, M. (RP144, RP145, RP146, RP147, RP148, RP150, RP151, RP153, RP158, RP16, RP166, RP184, RP185). 


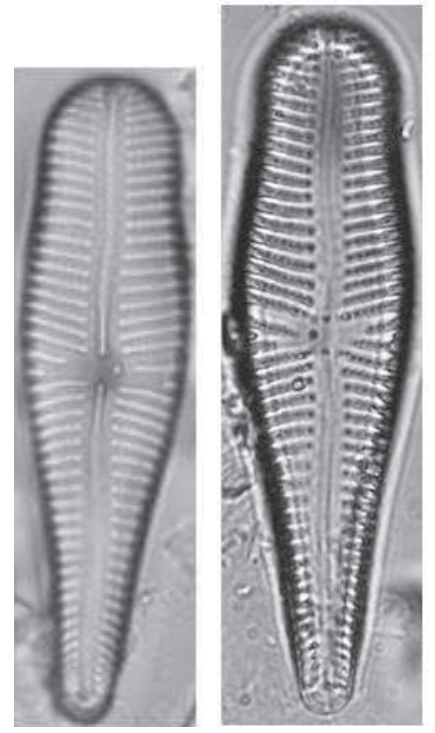

Figura 18 - Gomphonema laticollum Reichardt Studies on Diatoms, p. 199, pl. 5, figs. 1-14, 2001.

Valva clavada; intumescida na região mediana; constrição menos ou mais acentuada próximo da extremidade apical; ápice largamente arredondado a capitado-arredondado; extremidade basal atenuado-arredondada; área axial linear, estreita; área central irregular, delimitada pelo encurtamento irregular das estrias da região mediana; estigma isolado de um dos lados da valva; rafe sinuosa, extremidades proximais fletidas para o lado do estigma, dilatadas em poro; fissuras terminais da rafe em forma de gancho, fletidas para o lado oposto ao do estigma; 12 estrias transapicais em $10 \mu \mathrm{m}$, radiadas, irregularmente encurtadas na região mediana da valva; areolação conspícua, 16 aréolas em $10 \mu \mathrm{m}$; eixo apical: 42,8-45,5 $\mu \mathrm{m}$; eixo transapical: 9,4- 11,9 $\mu \mathrm{m}$.

Material examinado: BRASIL. Paraná: Guarapuava, rio das Pedras, 26/ III/2004, Bergamaschi, A.C. E Kawa, M. (RP007, RP020, RP024); Guarapuava, rio das Pedras, 30/IV/2004, Bergamaschi, A.C. E Kawa, M. (RP042); Guarapuava, rio das Pedras, 18/VI/2004, Bergamaschi, A.C. E Kawa, M. (RP113, RP123, RP131, RP132);
Guarapuava, rio das Pedras, 09/VIII/2004, Bergamaschi, A.C. E Kawa, M. (RP148, RP150, RP151, RP157, RP184, RP187).

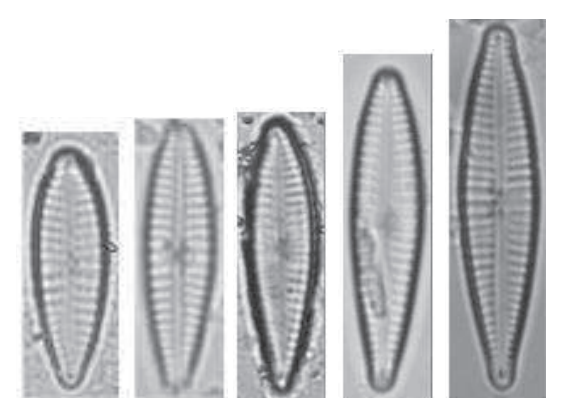

Figura 19 - Gomphonema parvulum (Kützing) Kützing Sp. Alg., p. 65. 1849.

Valva clavada, estreitamente lanceolada a elíptico-lanceolada; extremidade apical arredondada, sub-rostrada, rostrada a rostrada-capitada; extremidade basal atenuado-arredondada, sub-rostrada, rostrada a capitada; área central unilateral, formada pelo encurtamento de uma estria mediana; presença de estigma no lado oposto a estria encurtada; rafe central, com extremidades proximais curvadas para o lado do estigma e extremidades distais indistintas; 12 estrias transapicais em 10 $\mu \mathrm{m}$, indistintamente areoladas, levemente paralelas a radiadas na região mediana e radiadas no ápice e base; eixo apical: 24,3 $\mu \mathrm{m}$; eixo transapical: $5 \mu \mathrm{m}$.

Material examinado: BRASIL. Paraná: Guarapuava, rio das Pedras, 26/III/2004, Bergamaschi, A.C. E Kawa, M. (RP001, RP002, RP003, RP004, RP005, RP006, RP007, RP008, RP010, RP011, RP013, RP014, RP015, RP016, RP017, RP018, RP020, RP021, RP023, RP024); Guarapuava, rio das Pedras, 30/IV/2004, Bergamaschi, A.C. \& Kawa, M. (RP038, RP040, RP041, RP042, RP043, RP044, RP046, RP047, RP048, RP049, RP051, RP053, RP054); Guarapuava, rio das Pedras, 05/V/2004, Bergamaschi, A.C. E Kawa, M. (RP096, RP107, RP108); Guarapuava, rio das 
Pedras, 18/VI/2004, Bergamaschi, A.C. E Kawa, M. (RP110, RP112, RP113, RP114, RP115, RP116, RP118, RP122, RP123, RP124, RP127, RP128, RP129, RP130, RP131, RP132, RP135, RP136, RP137, RP139, RP141, RP142); Guarapuava, rio das Pedras, 09/VIII/2004, Bergamaschi, A.C. E Kawa, M. (RP145, RP146, RP147, RP148, RP149, RP150, RP152, RP153, RP154, RP157, RP158, RP166, RP187).

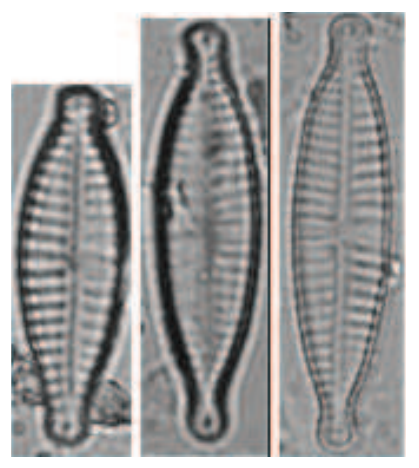

Figura 20 - Gomphonema lagenula Kützing Bacill., p. 85, pl. 30, fig. 60, 1844.

Valvas lanceoladas a elípticolanceoladas; ápices subcapitados a subrostrados e bases subcapitadas. Área axial linear e estreita com área central retangular, delimitada pelo encurtamento de uma estria mediana com estigma presente na base da estria mediana alongada no lado oposto; rafe reta, extremidades proximais fletidas para o lado em que o estigma está presente. 14-16 estrias em 10 $\mu \mathrm{m}$, unisseriadas, paralelas a levemente radiadas, as medianas mais afastadas das adjacentes. Aréolas inconspícuas. Eixo apical: $16,27-20,44 \mu \mathrm{m}$; eixo transapical: 5,16-7,44 $\mu \mathrm{m}$

Material examinado: BRASIL. Paraná: Guarapuava, rio das Pedras, 02/VIII/2004, Bergamaschi, A.C. \& Kawa, M. (RP144, RP148, RP150).

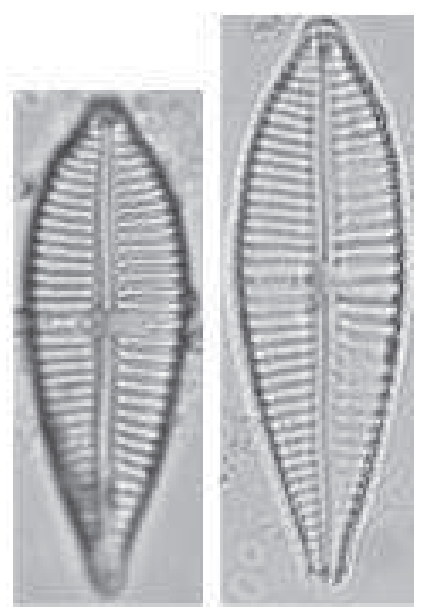

Figura 21 - Gomphonema pseudoaugur Lange-Bertalot Arch. Hydrobiol. 56(2): 213-214, figs. 11-16, 79, 80, 1979.

Valva lanceolada a elíptico-lanceolada; extremidade apical rostrada a sub-rostrada e extremidade basal atenuada, área axial linear, área central unilateral delimitada pelo encurtamento de uma estria mediana; estigma presente; rafe reta a levemente sinuosa, com extremidades proximais dilatadas em poro e fletidas para o lado em que o estigma está presente; extremidades distais da rafe fletidas para o lado oposto ao do estigma. 13-16 estrias em $10 \mu \mathrm{m}$, unisseriadas, paralelas a radiadas, mais espaçadas na região mediana, formadas por aréolas inconspícuas; eixo apical: 24,2-

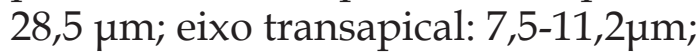

Material examinado: BRASIL. Paraná: Guarapuava, rio das Pedras, 26/III/2004, Bergamaschi, A.C. E Kawa, M. (RP006, RP008, RP009, RP017, RP018, RP022, RP023, RP024); Guarapuava, rio das Pedras, 30/IV/2004, Bergamaschi, A.C. E Kawa, M. (RP037, RP039, RP040, , RP043, RP047, RP053, RP055,); Guarapuava, rio das Pedras, 05/V/2004, Bergamaschi, A.C. \& Kawa, M. (RP096, RP105, RP107, RP108); Guarapuava, rio das Pedras, 18/ VI/2004, Bergamaschi, A.C. E Kawa, M. (RP110, RP112, RP114, RP116, RP117, RP122, RP123, RP124, RP125, RP126, RP127, RP128, RP131, RP132, RP135, 
RP136, RP137, RP139, RP141, RP142); Guarapuava, rio das Pedras, 09/VIII/2004, Bergamaschi, A.C. E Kawa, M. (RP144, RP148, RP150, RP151, RP153, RP158, RP166, RP184, RP185).

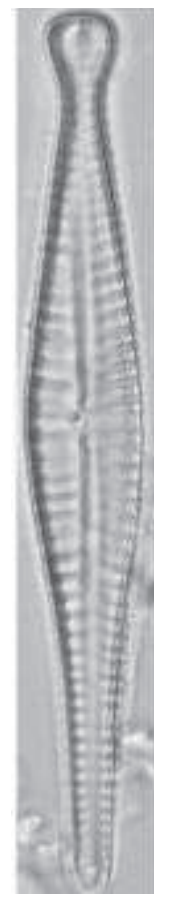

Figura 22 - Gomphonema subtile Ehrenberg Abh. Akad. Wiss. Berl., p. 416, 1841 (1843).

Valva heteropolar lanceolada; extremidade apical capitada; extremidade basal atenuado-arredondada; rafe filiforme, esterno da rafe linear, estreito; área central unilateral formada pelo encurtamento de uma estria mediana; estigma na extremidade da estria mediana; nove estrias radiadas em $10 \mu \mathrm{m}$; aréolas inconspícuas; eixo apical: $57,14 \mu \mathrm{m}$; eixo transapical: $7 \mu \mathrm{m}$.

Material examinado: BRASIL. Paraná: Guarapuava, rio das Pedras, 26/ III/2004, Bergamaschi, A.C. E Kawa, M. (RP007); Guarapuava, rio das Pedras, 18/VI/2004, Bergamaschi, A.C. E Kawa, M. (RP132).

\section{Ordem Naviculales Família Amphipleuraceae}

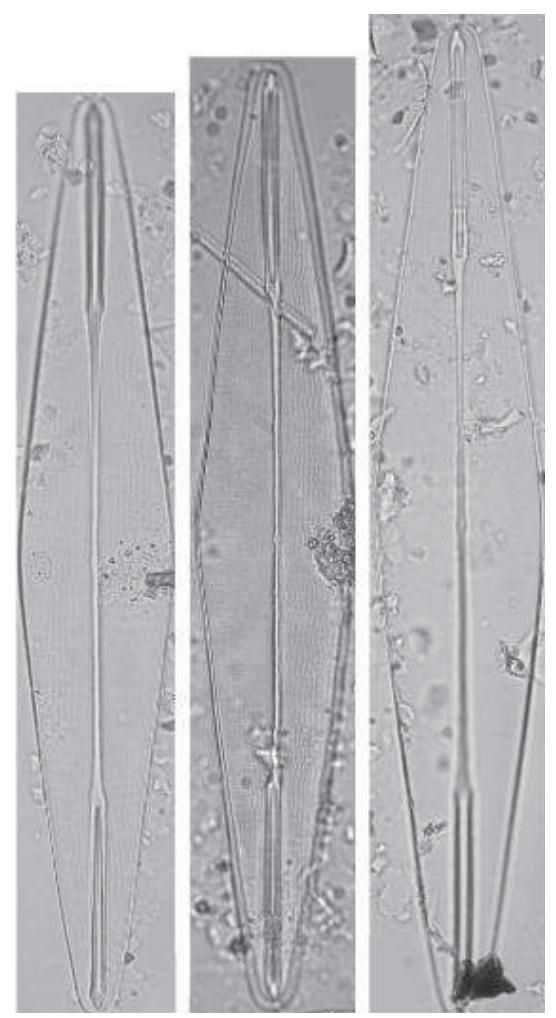

Figura 23 - Amphipleura lindheimerii Grunow Verh. Zool. Bot. Ges. Wein 12: 469, pl. 13, fig. 11a-b. 1862.

Valva rombo-lanceolada. Costelas silicosas entre as terminações da rafe; rafe linear estreita; comprimento de cada ramo da rafe varia de um terço a um sexto do comprimento da valva. 26-28 estrias paralelas em $10 \mu \mathrm{m}$, indistintamente pontuadas; linhas longitudinais onduladas, ou algumas vezes não perfeitamente numerosas como as estrias transversas; eixo apical: $122,7-183,2 \mu \mathrm{m}$; eixo transapical: 19,8-23,5 $\mu \mathrm{m}$.

Material examinado: BRASIL. Paraná: Guarapuava, rio das Pedras, 26/III/2004, Bergamaschi, A.C. E Kawa, M. (RP001, RP002, RP004, RP005, RP006, RP007, RP008, RP009, RP010, RP013, RP014, RP015, RP016, RP022, RP023, RP024); Guarapuava, rio das Pedras, 30/IV/2004, Bergamaschi, A.C. E Kawa, M. (RP038, RP042, RP043, RP047, RP048, RP051, 
RP057); Guarapuava, rio das Pedras, 18/ VI/2004, Bergamaschi, A.C. \& Kawa, M. (RP110, RP113, RP116, RP118, RP120, RP123, RP125, RP131, RP132, RP133, RP136); Guarapuava, rio das Pedras, 09/ VIII/2004, Bergamaschi, A.C. E Kawa, M. (RP150, RP151, RP157, RP159).

\section{Frustulia Rabenhorst}

Chave para os táxons de Frustulia

1. Rafe arqueada situada entre costelas levemente convexas Frustulia vulgaris

1. Rafe reta, contida entre duas costelas silicosas paralelas

2. Valvas com ondulações nas margens

2. Valvas sem ondulações nas margens

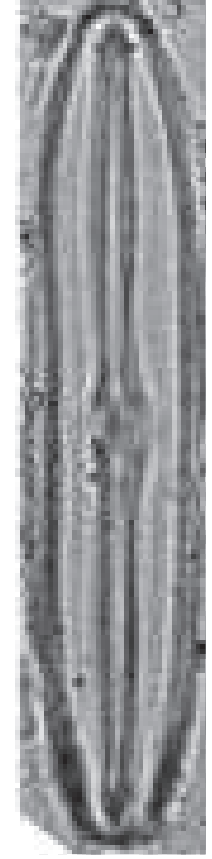

Figura 24 - Frustulia vulgaris (Thwaites) De Toni Syn. Alg. (2)1: 280. 1891.

Valva linear-lanceolada; extremidades rostradas; área axial linear, estreita; área central elíptica; rafe arqueada situada entre costelas levemente convexas; estrias inconspícuas, de difícil contagem, transversais radiadas na porção central; eixo apical: 30,6 $\mu \mathrm{m}$; eixo transapical: 5,9 $\mu \mathrm{m}$.
Material examinado: BRASIL. Paraná: Guarapuava, rio das Pedras, 18/VI/2004, Bergamaschi, A.C. E Kawa, M. (RP110, RP127); Guarapuava, rio das Pedras, 09/ VIII/2004, Bergamaschi, A.C. E Kawa, M. (RP157). 
Material examinado: BRASIL. Paraná: Guarapuava, rio das Pedras, 26/ III/2004, Bergamaschi, A.C. E Kawa, M. (RP001, RP004, RP005, RP006, RP014, RP016, RP017, RP018, RP023, RP024); Guarapuava, rio das Pedras, 30/IV/2004, Bergamaschi, A.C. E Kawa, M. (041, RP043, RP046, RP048, RP050, RP051, RP054, RP057); Guarapuava, rio das Pedras, 05/V/2004, Bergamaschi, A.C. E Kawa, M. (RP096, RP104, RP105, RP107, RP108); Guarapuava, rio das Pedras, 18/VI/2004, Bergamaschi, A.C. E Kawa, M. (RP110, RP112, RP121, RP122, RP127, RP128, RP129, RP130, RP131, RP133 RP136, RP138, RP139, RP140, RP142); Guarapuava, rio das Pedras, 09/ VIII/2004, Bergamaschi, A.C. \& Kawa, M. (RP148, RP149, RP150, RP153, RP154, RP58, RP185).

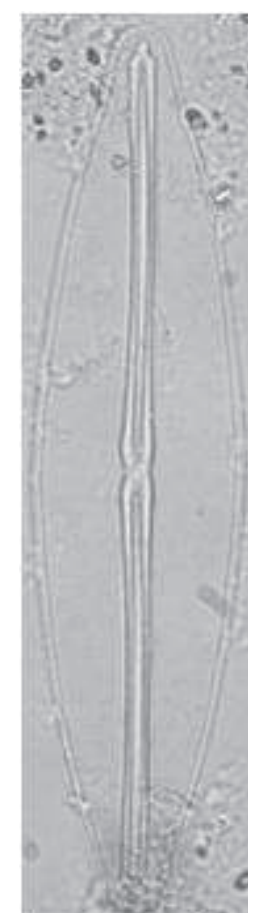

Figura 26 - Frustulia saxonica Rabenhorst

Süssw.-Diat. Freund. Mikrosk., p. 50, pl. 7, fig. 1, 1853.

Valvas lanceoladas; extremidades subrostradas; área axial linear, estreita; área central constricta, rafe reta, contida entre duas costelas silicosas paralelas; 17 estrias transversais, paralelas e 22 linhas hialinas longitudinais levemente onduladas em 10 $\mu \mathrm{m}$, muito finas. Eixo apical: $81 \mu \mathrm{m}$; eixo transapical: $18,2 \mu \mathrm{m}$; estrias: $30 \mathrm{em} 10 \mu \mathrm{m}$; linhas longitudinais: $24 \mathrm{em} 10 \mu \mathrm{m}$.

Material examinado: BRASIL. Paraná: Guarapuava, rio das Pedras, 26/III/2004, Bergamaschi, A.C. E Kawa, M. (RP002, RP008, RP012, RP013, RP020, RP021, RP022); Guarapuava, rio das Pedras, 30/ IV/2004, Bergamaschi, A.C. E Kawa, M. (RP039, RP040, RP046, RP047, RP048, RP050, RP051, RP054); Guarapuava, rio das Pedras, 05/V/2004, Bergamaschi, A.C. \& Kawa, M. (RP096, RP104, RP108); Guarapuava, rio das Pedras, 18/VI/2004, Bergamaschi, A.C. E Kawa, M. (RP110, RP112, RP113, RP114, RP117, RP118, RP119, RP121, RP122, RP124, RP136, RP137, RP138, RP141, RP142); Guarapuava, rio das Pedras, 09/VIII/2004, Bergamaschi, A.C. \& Kawa, M. (RP143, RP144, RP145, RP146, RP151, RP153, RP154, RP157, RP58).

\section{Família Diadesmidaceae}

\section{Diadesmis Kützing}

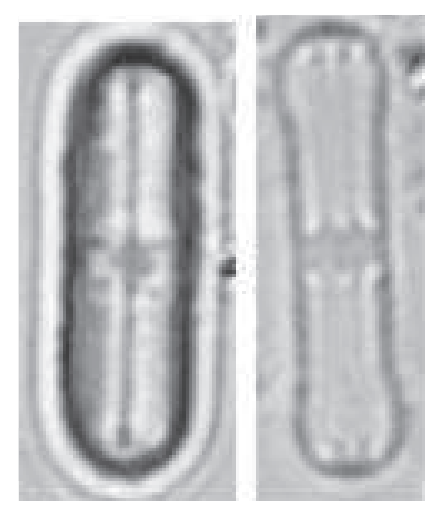

Figura 27 - Diadesmis contenta (Grunow ex Van Heurck) D.G. Mann Round, Crawford \& Mann. The Diatoms, p. 666. 1990. 
Valvas lineares, por vezes levemente constrictas na região mediana; extremidades largamente arredondadas; rafe linear reta; área central retangular; estrias de difícil visualização; eixo apical: 8-10,3 $\mu \mathrm{m}$; eixo transapical: 2-3 $\mu \mathrm{m}$.

Material examinado: BRASIL. Paraná: Guarapuava, rio das Pedras, 26/III/2004, Bergamaschi, A.C. E Kawa, M. (RP001, RP003, RP015, RP018, RP023); Guarapuava, rio das Pedras, 30/IV/2004, Bergamaschi, A.C. E Kawa, M. (RP038, RP044, RP046, RP048, RP051, RP052); Guarapuava, rio das Pedras, 05/V/2004, Bergamaschi, A.C. $\mathcal{E}$ Kawa, M. (RP096, RP104); Guarapuava, rio das Pedras, 18/VI/2004, Bergamaschi, A.C. E Kawa, M. (RP109, RP112, RP116, RP117, RP123, RP127, RP128, RP131, RP133, RP135, RP136, RP137, RP142); Guarapuava, rio das Pedras, 09/VIII/2004, Bergamaschi, A.C. E Kawa, M. (RP143, RP144, RP146, RP147, RP149, RP152, RP153, RP154, RP157, RP159).

\section{Luticola D.G. Mann}

Chave para os táxons de Luticola

1. Valva com margens não onduladas

1. Valva com margens onduladas

2. Área central retangular

2. Área central linear

Luticola undulata

Figura 28 - Luticola dapalis (Frenguelii) D.G. Mann Round, Crawford \& Mann. The Diatoms, p. 670.1990.

Luticola muticoides

3. Ausência de linha longitudinal submarginal

3. Presença de uma linha longitudinal submarginal

4. Eixo apical: $13-20 \mu \mathrm{m}$

Luticola dapalis

4. Eixo trasapical: $20-32,5 \mu \mathrm{m}$ Luticola mutica

Valva lanceoladas com margens não onduladas; extremidades rostradas; área axial lanceolada; área central retangular, com estrias encurtadas e presença de estigma alongado; rafe filiforme, com extremidades proximais voltadas para o lado oposto ao estigma; 12 estrias transapicais em $10 \mu \mathrm{m}$, nitidamente pontuadas, 12 aréolas em $10 \mu \mathrm{m}$; presença de uma linha longitudinal submarginal; eixo apical: 73,5 $\mu \mathrm{m}$; eixo transapical: 24,2 $\mu \mathrm{m}$.

Material examinado: BRASIL. Paraná: Guarapuava, rio das Pedras, 26/III/2004, Bergamaschi, A.C. E Kawa, M. (RP016, RP023); Guarapuava, rio das Pedras, 09/ VIII/2004, Bergamaschi, A.C. E Kawa, M. (RP159). 


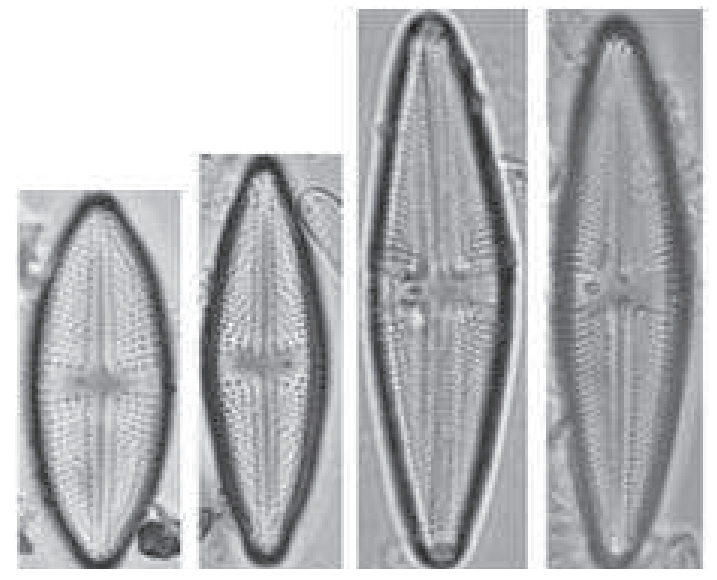

Figura 29 - Luticola goeppertiana (H.L. Smith) D.G. Mann Round, Crawford \& Mann. The Diatoms, p. 670.1990.

Valva elíptica a lanceolada; extremidades arredondadas a sub-rostradas; área axial linear a levemente lanceolada; área central retangular, circundada por estrias encurtadas; presença de estigma isolado, às vezes alongado; rafe reta, com extremidades proximais voltadas para direções contrárias ao estigma; 18-22 estrias transapicais distintamente areoladas, 18 aréolas em $10 \mu \mathrm{m}$, radiadas ao logo da superfície valvar; eixo apical: 20-32,5 $\mu \mathrm{m}$; eixo transapical: 7,3-8,5 $\mu \mathrm{m}$.

Material examinado: BRASIL. Paraná: Guarapuava, rio das Pedras, 26/III/2004, Bergamaschi, A.C. E Kawa, M. (RP001, RP013, RP016); Guarapuava, rio das Pedras, 30/IV/2004, Bergamaschi, A.C. E Kawa, M. (RP038, RP040, RP043, RP046, RP051); Guarapuava, rio das Pedras, 18/ VI/2004, Bergamaschi, A.C. E Kawa, M. (RP114, RP118, RP125, RP127, RP128, RP136, RP137, RP142); Guarapuava, rio das Pedras, 09/VIII/2004, Bergamaschi, A.C. E Kawa, M. (RP143, RP146, RP147, RP149, RP150, RP152, RP159).

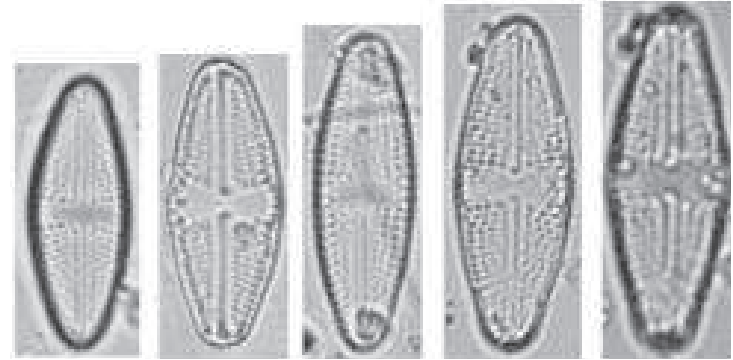

Figura 30 - Luticola mutica (Kützing) D.G. Mann Round, Crawford \& Mann. The Diatoms, p. 670. 1990.

Valva elíptico-lanceolada a rômbicalanceolada; extremidades arredondadas a sub-rostradas; área axial linear a levemente lanceolada; área axial linear, estreito; área central retangular, podendo atingir as margens valvares; presença de estigma isolado, às vezes alongado; rafe filiforme, com extremidades proximais retas a fracamente curvas voltadas para direções contrárias ao estigma; 15-17 estrias transapicais distintamente areoladas em 10 $\mu \mathrm{m}$, radiadas ao logo da superfície valvar; eixo apical: 13,4-20,9 $\mu$ m; eixo transapical: 5,7-8 $\mu \mathrm{m}$.

Material examinado: BRASIL. Paraná: Guarapuava, rio das Pedras, 26/III/2004, Bergamaschi, A.C. E Kawa, M. (RP002, RP019); Guarapuava, rio das Pedras, 30/ IV/2004, Bergamaschi, A.C. E Kawa, M. (RP039, RP041, RP043, RP046, RP051, RP054); Guarapuava, rio das Pedras, 05/V/2004, Bergamaschi, A.C. E Kawa, M. (RP104, RP107, RP108); Guarapuava, rio das Pedras, 18/VI/2004, Bergamaschi, A.C. $\mathcal{E}$ Kawa, M. (RP110, RP112, RP113, RP114, RP117, RP122, RP125, RP127, RP136, RP137, RP141); Guarapuava, rio das Pedras, 09/VIII/2004, Bergamaschi, A.C. E Kawa, M. (RP144, RP151, RP152, RP153). 


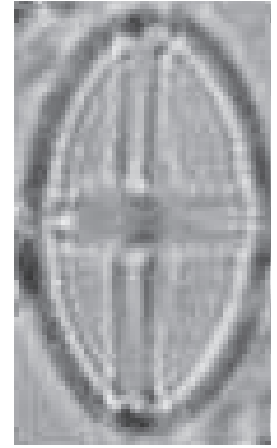

Figura 31 - Luticola muticoides (Hustedt) D.G. Mann

Round, Crawford \& Mann. The Diatoms, p. 671, 1990.

Valva elíptica a elíptico-lanceolada; extremidades arredondadas; esterno da rafe linear, área central linear, alcançando as margens da valva; estigma linear, marginal; rafe filiforme; 18 estrias radiadas em $10 \mu \mathrm{m}$; aréolas delicadas, arredondadas; eixo apical: $15 \mu \mathrm{m}$; eixo transapical: $8 \mu \mathrm{m}$.

Material examinado: BRASIL. Paraná: Guarapuava, rio das Pedras, 26/III/2004, Bergamaschi, A.C. E Kawa, M. (RP015).

\section{Família Neidiaceae}

Neidium Pfitzer

Chave para os táxons de Neidium

1. Valva amplamente linear a linear-elíptica, banda longitudinal submarginal presente ...

1. Valva linear, banda longitudinal submarginal ausente

Neidium affine Neidium catarinense 


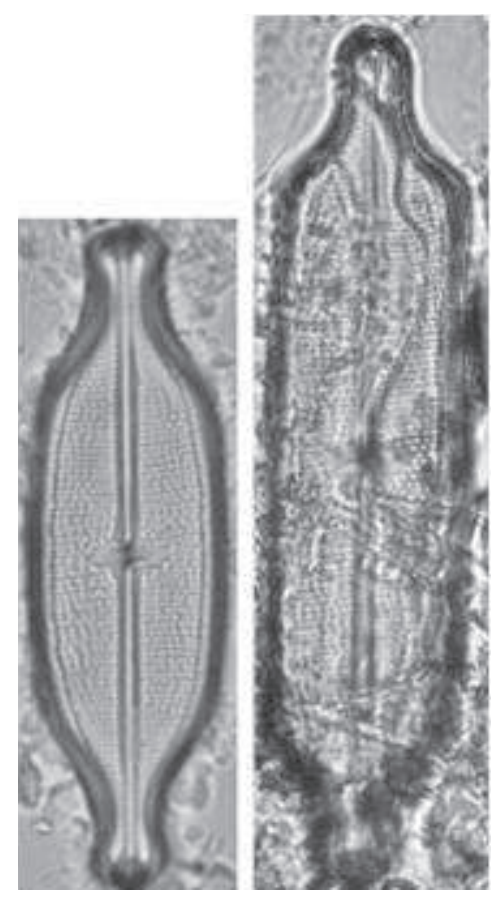

Figura 33 - Neidium affine (Ehrenberg) Pfitzer Bot. Abh. Geb. Morph. Physiol., v. 1, n. 2, p. 39, 1871.

Valva amplamente linear a linearelíptica; extremidades longas, rostradas a capitadas, cerca de um terço do tamanho da valva a partir do centro; área axial reta, mais estreita perto da área central; nódulos terminais evidentes; rafe reta, estreitando-se perto das terminações proximais e distais; terminações proximais curvadas em direções opostas; terminações distais bifurcadas; área central pequena, diagonalmente elíptica; banda longitudinal primária presente, submarginal. 20-23 estrias em $10 \mu \mathrm{m}$, mais ou menos oblíquas, exceto nas terminações onde elas são paralelas a convergentes; eixo apical: 43,657,6 $\mu \mathrm{m}$; eixo transapical: 12,4-13,4 $\mu \mathrm{m}$.

Material examinado: BRASIL. Paraná: Guarapuava, rio das Pedras, 26/III/2004, Bergamaschi, A.C. E Kawa, M. (RP002, RP013, RP023); Guarapuava, rio das Pedras, 30/IV/2004, Bergamaschi, A.C. $\mathcal{E}$ Kawa, M. (RP057); Guarapuava, rio das Pedras, 18/VI/2004, Bergamaschi, A.C. \& Kawa, M. (RP114, RP136, RP141);
Guarapuava, rio das Pedras, 09/VIII/2004, Bergamaschi, A.C. E Kawa, M. (RP145, RP146, RP150).

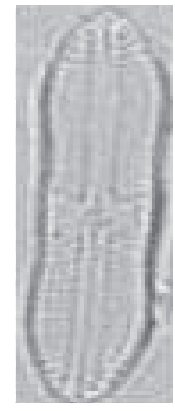

Figura 34 - Neidium catarinense (Krasske) Lange-Bertalot Iconogr. Diatomol., v. 3, p. 100-101, pl. 31, figs. 1-2, 1996.

Valva linear com margens trionduladas; extremidades cuneado-arredondadas; esterno da rafe linear, estreito; área central quadrangular; rafe filiforme, terminações proximais curvadas em direções opostas; 18 estrias radiadas em $10 \mu \mathrm{m}$; banda longitudinal primária submarginal ausente; aréolas inconspícuas; eixo apical: 19,28 $\mu \mathrm{m}$; eixo transapical: 5,7 $\mu \mathrm{m}$.

Material examinado: BRASIL. Paraná: Guarapuava, rio das Pedras, 26/III/2004, Bergamaschi, A.C. \& Kawa, M. (RP002).

\section{Ordem Thalassiophysales Família Catenulaceae}

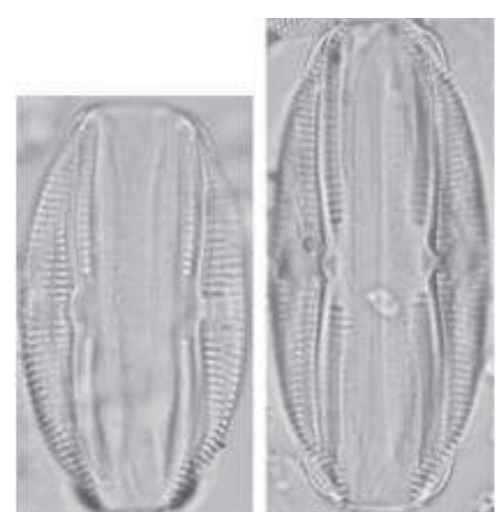

Figura 35 - Amphora ovalis (Kützing) Kützing Die kiesels. Bacill. Diat., p. 1-6, [1]-152, pls 1-30. 1844. 
Valva dorsiventral; extremidades arredondadas em bico; sistema da rafe ao longo da linha mediana da valva. 13 estrias unisseriadas com aréolas alongadas; eixo apical: 36,2-45 $\mu$ m; eixo transapical: 20-20,6 $\mu \mathrm{m}$.

Material examinado: BRASIL. Paraná: Guarapuava, rio das Pedras, 26/III/2004, Bergamaschi, A.C. E Kawa, M. (RP005, RP007, RP009).

\section{CONCLUSÕES}

Foram considerados táxons constantes: Frustulia saxonica Rabenhorst, Gomphonema gracile Ehrenberg e Gomphonema parvulum (Kützing) Kützing com frequências superiores a $50 \%$ das amostras. Esse último táxon, que obteve maior frequência, é citado na literatura como uma espécie característica de águas muito poluídas por matéria orgânica, chegando a ser abundante nesse ambiente. Frustulia saxonica Rabenhorst e Gomphonema gracile Ehrenberg são referidas na literatura como espécies oligohalóbias epífitas, que são aquelas espécies muito difundidas em água doce e que vivem em ambientes com limites salinos muito restritos.

Os índices ecológicos apresentaram os seguintes resultados: índice halóbio predominam qualitativamente as espécies oligohalóbias indiferentes, espécies que vivem em água doce, mas podem suportar pequenas alterações salinas no ambiente; índice sapróbico: predominaram espécies oligossapróbias com tendência a mesossapróbias, desenvolvendo-se em ambiente com decomposição de matéria orgânica (proteínas, amônia, sais, etc.); com relação ao nível trófico, as espécies eutróficas predominaram, revelando águas ricas em substâncias nutritivas.

A análise da comunidade diatomológica presente no rio das Pedras, Guarapuava,
Paraná, forneceu informações das condições ecológicas, sendo que a predominância de espécies eutróficas revela águas ricas em substâncias nutritivas, indicando que o sistema encontra-se alterado devido ao efeito causado por início de degradação de compostos orgânicos lançados no corpo d'água.

\section{REFERÊNCIAS}

BATTISTELLI, M.; CAMARGO FILHO, M.; HEERDT, B. (Org.). Proteção e manejo da bacia do rio das Pedras: relato de experiências. Brasília: B\&D, 2004. 134 p.

BRASSAC, N.M.; LUDWIG, T.V. Amphipleuraceae e Diploneidaceae (Bacillariophyceae) da bacia do rio Iguaçu, PR, Brasil. Acta Botanica Brasilica, v. 19, n. 2, p. 359-368, 2005.

CETTO, J.M.; LEANDRINI, J.A.; FELISBERTO, S.A.; RODRIGUES, L. Comunidade de algas perifíticas no reservatório de Iraí, Estado do Paraná, Brasil. Acta Scientiarum. Biological Sciences, v. 26, n. 1, p. 1-7, 2004.

FERRARI, F. ; LUDWIG, T.A.V. Coscinodiscophyceae, Fragilariophyceae e Bacillariophyceae (Achnanthales) dos rios Ivaí, São João e dos Patos, bacia hidrográfica do rio Ivaí, município de Prudentópolis, PR, Brasil. Acta Botanica Brasilica, v. 21, n. 2, p. 421-441, 2007.

HUSTEDT, F. Bacillariophyta (Diatomeas). In: PASCHER, A. Die Süswasser-Flora Mitteleuropas. 2ed. Jena, G. Fischer, 1930. v. 10, 446p.

HUSTEDT, F. Die Kieselalgen. In: RABENHORST'S, L. Kriptogamen-Flora von Deustchland, Österreich und der Schweiz. Leipizig: Akademische Verlagsgesellschaft m.b.H., 1927-30.v.7, pt. 1,920p.

HUSTEDT, F. Systematische und ökologische Untersuchungen über die Diatomeen-Flora von Java, Bali und Sumatra. Archiv für Hydrobiol., v. 16, p. 274-394, 1939.

HUSTEDT, F. Die Kieselalgen. In: RABENHORST'S, L. Kriptogamen-Flora von Deustchland, Österreich und der Schweiz. Leipizig: Akademische Verlagsgesellschaft m.b.H., 1961-66. v. 7, pt. 3, 816p.

HUSTEDT, F. Neue und wenig bekannte Diatomeen. III. Phylogenetische Variationen bei 
den rhaphidioiden Diatomeen. Ber. Deutsch. Bot. Ges., v. 65, p. 133-144, 1952.

HUSTEDT, F. Neue und wenig bekannte Diatomeen. IX. Süswasserdiatomeen aus Brasilien, insbesondere des Amazonasgebietes. Int. Rev. Ges. Hydrobiol., v. 50, n. 3, p. 391-410, 1965.

KRAMMER, K., LANGE-BERTALOT, H. Bacillariophyceae: Naviculaceae. In: ETTL, H.; GERLOFF, J.; HEYNIG, H.; MOLLENHAUER, D. Süswasserflora von Mitteleuropa. Stuttgart: G. Fischer. 1986, v. 2, pt. 1, 876p.

KRAMMER, K., LANGE-BERTALOT, H. Bacillariophyceae: Bacillariaceae, Epithemiaceae, Surirellaceae. In: ETTL, H.; GERLOFF, J.; HEYNIG, H.; MOLLENHAUER, D. Süswasserflora von Mitteleuropa. Stuttgart: G. Fischer, 1988, v. 2, pt. 2, 596p.

KRAMMER, K., LANGE-BERTALOT, H. Bacillariophyceae: Centrales, Fragilariaceae, Eunotiaceae. In: ETTL, H.; GERLOFF, J.; HEYNIG, H.; MOLLENHAUER, D. Süswasserflora von Mitteleuropa. Stuttgart: G. Fischer, 1991a, v. 2, pt. 3, 576p.

KRAMMER, K., LANGE-BERTALOT, H. Bacillariophyceae: Achnanthaceae. Kritische Ergänsungen zu Navicula (Lineolatae) und Gomphonema. In: ETTL, H.; GERLOFF, J.; HEYNIG, H.; MOLLENHAUER, D. Süswasserflora von Mitteleuropa. Stuttgart: G. Fischer, 1991b, v. 2, pt. 4, 437p.

KRAMMER, K. Bibliotheca Diatomologica: Die cymbelloiden Diatomeen: eine monographie der weltweit bekannten taxa. II. Encyonema Part., Encyonopsis and Cymbellopsis. v. 37, Berlin: J. Cramer, 1997, 469p.

KRUPEK, R. A. Estudo das comunidades de macroalgas da Bacia do Rio das Pedras, Guarapuava, Paraná: Flora e dinâmica sazonal. Curitiba, 2006. Dissertação (Mestrado em Botânica) - Universidade Federal do Paraná.

LEANDRINI, J.A. Diatomáceas perifíticas da região de captação de água da SANEPAR, Maringá-PR. Curitiba, 1999. Dissertação (Mestrado em Botânica) - Universidade Federal do Paraná.

MOREIRA-FILHO, H.; VALENTE-MOREIRA, I. M. Avaliação taxonômica e ecológica das diatomáceas (Bacillariophyceae) epífitas em algas pluricelulares obtidas nos litorais dos estados do Paraná, Santa Catarina e São Paulo. Boletim do Museu Botânico Municipal de Curitiba, n. 47, p. 1-17, 1981.
PATRICK, R.; REIMER, C.W. The diatoms of the United States. Philadelphia, Academy of Natural Sciences, 1966. v.1. 668p.

PATRICK, R.; REIMER, C.W. The diatoms of the United States. Philadelphia, Academy of Natural Sciences, 1975. v.2. 213p.

PIECZYNSKA, E. Periphyton in the trophic structure os freshwater ecosystems. Pol. Arch. Hydrobiol., v.17 (1, 2), p. 141-147, 1970.

ROUND, F.E.; CRAWFORD, R.M.; MANN, D.G. The diatoms: Biology and Morphology of the Genera. Cambridge: Cambridge University Press, 1990. 747p.

SANTOS, A.F.; KOBIYAMA, M. Contribuição potencial de cargas poluentes na bacia do Rio das Pedras, no município de Guarapuava -PR. Rev. Ciências Exatas e Naturais, n. 5, p. 33-46, 2003.

SLÁDECEK, V.; SLÁDECKOVÁ, A. Relationship between wet weight and dry weight of the periphyton. Limnol. Oceanogr., v. 8, n. 2, p. 309-311, 1963.

SLÁDECEK, V. The indicator value of some freemoving ciliates. Arch. Protistenk. Bd., v. 111, p. 276278, 1969.

SLÁDECKOVÁ, A.; SLÁDECEK, V. Periphyton as indicator of the reservoir water quality II. Pseudoperiphyton. Arch. Hydrobiol. Belh. Ergerbn. Limnol., v. 9, p. 177-191, 1977.

SLÁDECKOVÁ, A.; MARVAN, P.; VYMAZAL, J. The utilization of periphyton in waterworks pretreatment for nutrient removal from enriched influents. In: WETZEL, R.E. (Ed.). Periphyton of freswater ecosystems. The Hague Dr. W. Junk, p.299-303. (Developments in Hydrobiology, 17), 1983.

SLÁDECKOVÁ, A.the role of periphyton in waste treatment technology. Verh. Internat. Verein. Limnol., v. 25, p. 1929-1932, 1994a.

SLÁDECKOVÁ, A. Green algae and treatment technology. Biologia, Bratislava. v. 49. p. 615-619, 1994b.

WATANABE, T. Etude de la relation entre le periphyton et la qualité chimique de Peau des rivieres: utilization de bioessais "in situ" (subsatrates artificiales) pour caracteriser Pétat de pollution des eaux. Tolouse/França: Université Paul Sabatier de Tolouse, $1985.127 \mathrm{p}$.

WATANABE, T.; ASKAI, K.;HOUKI, A. Numerical estimation to organic pollution of lowing water by using the epilithic diatom assemblage- Diatom 
Gisele Carolina Marquardt et AL.

assemblage Index (DAI). The Science of the total Environment. v. 55. p. 209-218, 1986a.

WATANABE, T.; ASKAI, K. \& HOUKI, A. Numerical water quality monitoring of organic pollution using diatom assemblage. $9^{\text {th }}$ Diatom Symposium. p. 123141, 1986b.

WATANABE, T. Perifiton: comparação de metodologias empregadas para caracterizar o nível de poluição das águas. Acta Limnol. Brasil., v. 3. p. 593-615, 1990.

Recebido em: 23/12/10

Aceito em: 19/03/11 